\title{
Materials informatics based on evolutionary algorithms: Application to search for superconducting hydrogen compounds
}

\author{
Takahiro Ishikawa $\odot,{ }^{1,2, *}$ Takashi Miyake $\odot,{ }^{3}$ and Katsuya Shimizu ${ }^{2}$ \\ ${ }^{1}$ ESICMM, National Institute for Materials Science, 1-2-1 Sengen, Tsukuba, Ibaraki 305-0047, Japan \\ ${ }^{2}$ Center for Science and Technology under Extreme Conditions, Graduate School of Engineering Science, \\ Osaka University, 1-3 Machikaneyama, Toyonaka, Osaka 560-8531, Japan \\ ${ }^{3}$ CD-FMat, National Institute of Advanced Industrial Science and Technology, 1-1-1 Umezono, Tsukuba, Ibaraki 305-8568, Japan
}

(Received 2 August 2019; revised manuscript received 9 October 2019; published 6 November 2019)

\begin{abstract}
We present a materials informatics approach to search for superconducting hydrogen compounds, which is based on a genetic algorithm and a genetic programing. This method consists of five stages: (i) collection of physical and chemical property data, (ii) development of superconductivity predictor based on the collected data by a genetic programing, (iii) prediction of potential candidates for high temperature superconductivity by regression analysis, (iv) crystal structure search of the candidates by a genetic algorithm, and (v) validation of the superconductivity by first-principles calculations. By repeatedly performing the process as (i) $\rightarrow$ (ii) $\rightarrow$ (iii) $\rightarrow$ (iv) $\rightarrow$ (v) $\rightarrow$ (i) $\rightarrow \ldots$, the database and predictor are further improved, which leads to an efficient search for superconducting materials. Using the first-principles data of binary hydrogen compounds, many of which have not been experimentally realized yet, we applied this method to hypothetical ternary ones and predicted $\mathrm{KScH}_{12}$ with a modulated hydrogen cage showing the superconducting critical temperature of $122 \mathrm{~K}$ at $300 \mathrm{GPa}$ and $\mathrm{GaAsH}_{6}$ showing $98 \mathrm{~K}$ at $180 \mathrm{GPa}$.
\end{abstract}

DOI: 10.1103/PhysRevB.100.174506

\section{INTRODUCTION}

The use of an informatics approach to materials science, i.e., materials informatics (MI), has been expected to bring the acceleration for the exploration of new materials [1-4]. High-throughput screening and machine learning have been employed to discover hidden trends and create predictive models in databases of physical and chemical properties for crystalline compounds, e.g., search for cathode materials with a long cycle life for lithium-ion battery [5] and new superconducting materials [6].

The search for new physical and chemical properties has been performed by varying compositions and external parameters such as pressure, temperature, and electromagnetic field with respect to already known materials. These approaches are considered as the exploration of optimal solutions in vast and complicated search space under given conditions. In such a case, evolutionary algorithm (EA), which is a heuristic-based approach to solving problems using mechanisms inspired by biological evolution, e.g., mating, mutation, selection, inheritance, etc., is effective for the discovery of the optimal solutions. EA has been used in a wide variety of scientific research fields and has been applied to search for stable or metastable crystal structures [7-13], well-performed mathematical equations and computer programs in predefined task, and so on.

In this study, we propose a materials informatics approach to search for new functional materials, which is based on a genetic algorithm (GA) and a genetic programing (GP)

*Ishikawa.Takahiro@nims.go.jp included in the EA techniques (Fig. 1). This method consists of five stages: (i) collection of physical and chemical property data (data collection), (ii) development of a functionality predictor based on the collected data by GP (GP training), (iii) prediction of potential candidates for high functionality by regression analysis (GP prediction), (iv) crystal structure search of the candidates by GA (GA structure search), and (v) validation of the functionality by first-principles calculations (first-principles validation). By repeatedly performing the process as (i) $\rightarrow$ (ii) $\rightarrow$ (iii) $\rightarrow$ (iv) $\rightarrow$ (v) $\rightarrow$ (i) $\rightarrow \ldots$, the database and predictor are gradually improved using the validation results. Thus this method enables one to accelerate the search for new functional materials. This paper is organized as follows: The details of GP for predictor development and GA for crystal structure search are presented in Sec. II, the application of our MI method to the search for superconducting hydrogen compounds is shown in Sec. III, and the discussion and conclusion are drawn in Sec. IV.

\section{EVOLUTIONARY ALGORITHMS}

Figure 2 shows a process on the search for high-quality optimal solutions by EA. First, we prepare for a population consisting of individuals $(I)$, which are generated randomly and ranked according to a fitness parameter (zeroth generation). Then, few especially inferior individuals are eliminated from the population (elimination), and the other ones are used for the creation of new individuals for the next generation. The new individuals are created by randomly applying evolutionary operations, "mating" and "mutation." Few especially superior individuals are inherited to the next generation (inheritance), and all the individuals are ranked 


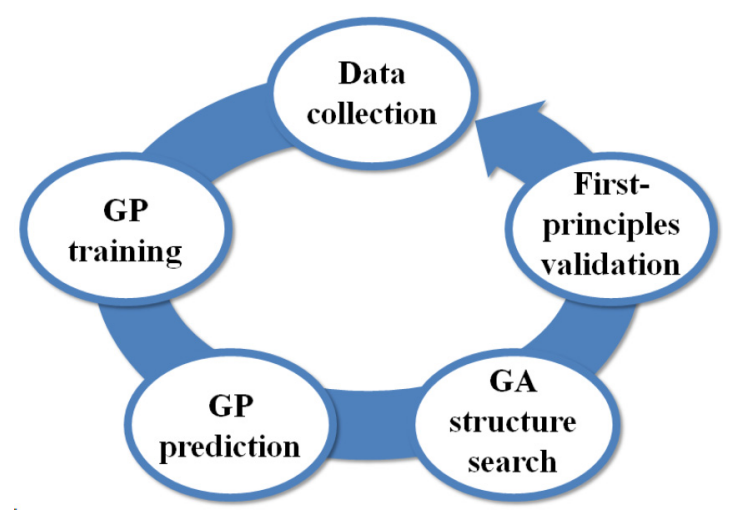

FIG. 1. Materials informatics approach to search for new functional materials, which is based on the genetic algorithm (GA) and the genetic programing (GP).

again. By repeatedly performing this process, the population is evolved to a more superior one and finally high-quality optimal solutions are obtained. As mentioned above, in our MI approach, we use GP to develop a functionality predictor and GA to search for stable crystal structures.

\section{A. GP for predictor development}

Figure 3 shows a flowchart at each generation with respect to the development of the predictor by GP. In the GP search, individuals are represented as computer programs or mathematical functions using tree structures as shown in Fig. 4. The tree structure consists of branch and leaf nodes and, in our GP search, a branch node depicts an element from arithmetic operators $(+,-, \times, \div)$ and a leaf node depicts an element from constants (1-10) and variables to develop mathematical functions. For example, the left tree of Fig. 4(a) represents the function of $f(A, B)=(2 \times A)+(3 \div B)$, which is written as $f(A, B)=+\times 2 A \div 3 B$ in the Polish notation to deal with arithmetic expression in programing language. The mating is the operator to create new tree structures by exchanging branches between two individuals selected randomly [Fig. 4(a)] and the mutation is the operator to exchange branches within an individual [Fig. 4(b)]. The individuals are ranked according to the strength of the correlation between the value obtained by substituting the data sets into the function (evaluation value: $x$ ) and physical property data which we will

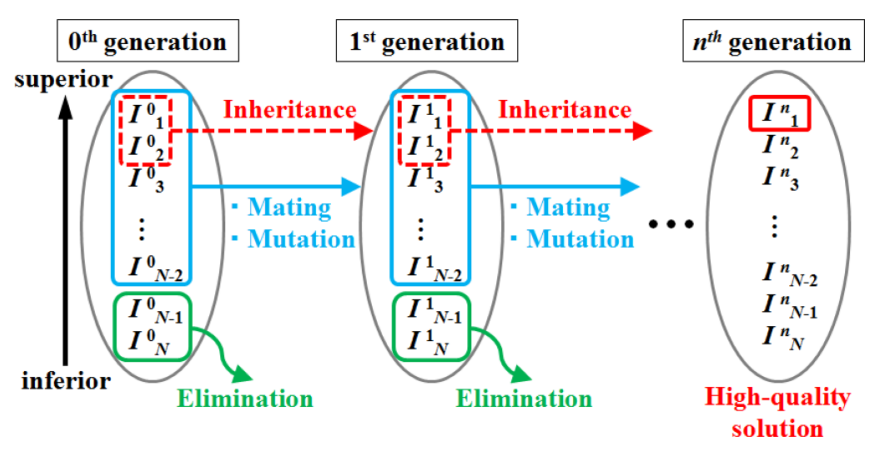

FIG. 2. Schematic view of evolutionary algorithm. $I$ represents an individual and $N$ is the number of individuals in the population.

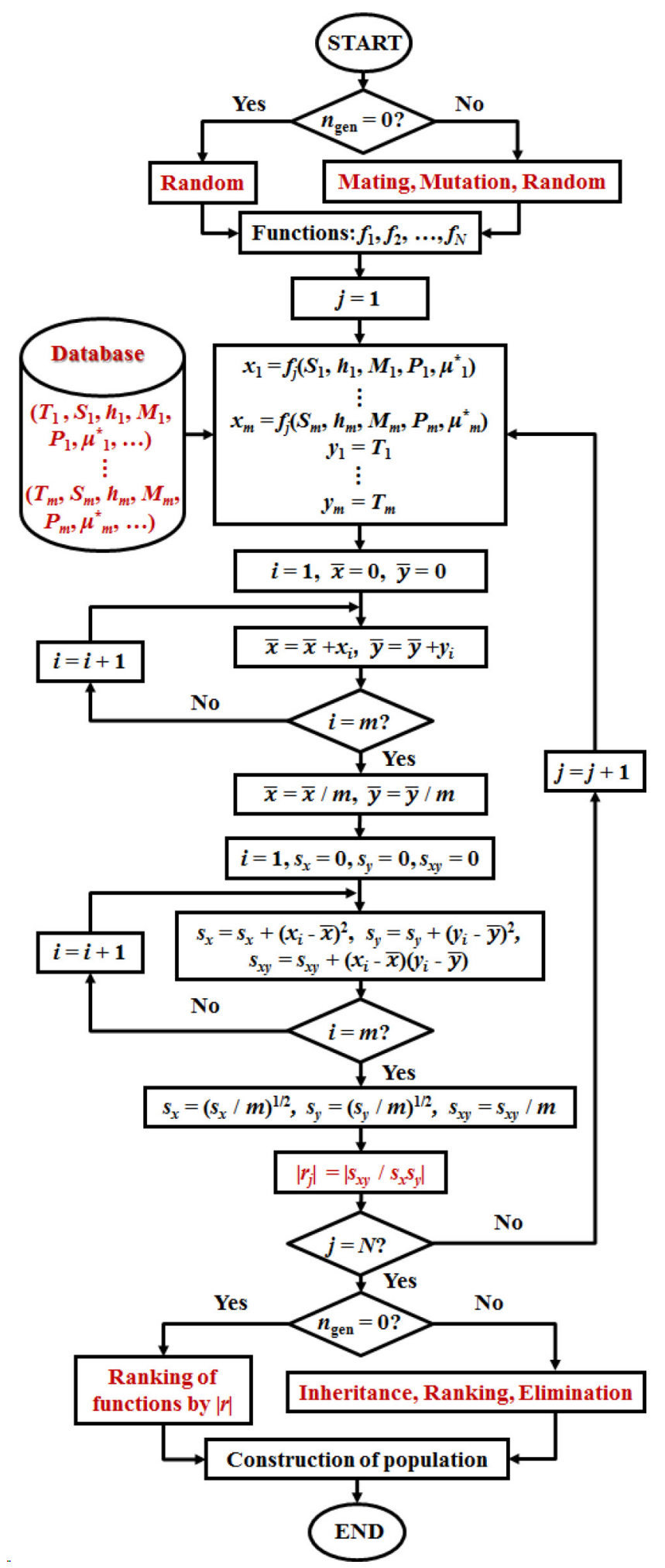

FIG. 3. Flowchart at each generation with respect to functionality predictor development by GP. The case of the superconductivity is shown as an example of the functionality (see Sec. III B).

investigate $(y)$. The absolute value of correlation coefficient ( $r$ ) between $x$ and $y$ is calculated by $|r|=\left|s_{x y} / s_{x} s_{y}\right|$, using the data included in the database. The parameters $s_{x}, s_{y}$, and $s_{x y}$ 
(a)
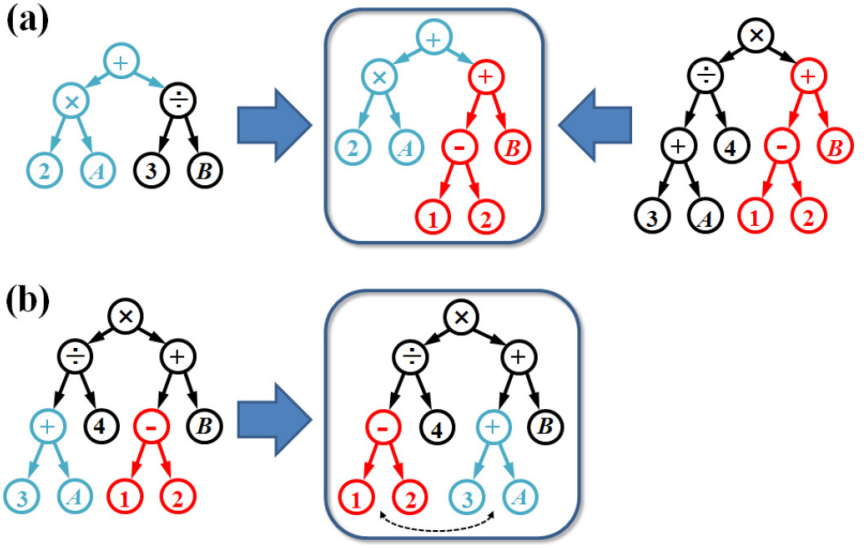

FIG. 4. Schematic view of mathematical function development by evolutionary operations: (a) mating and (b) mutation.

are a standard deviation of data $x$, that of $y$, and a covariance between $x$ and $y$, respectively. They are calculated as follows:

$$
\begin{aligned}
& s_{x}=\left(\frac{1}{m} \sum_{i=1}^{m}\left(x_{i}-\bar{x}\right)^{2}\right)^{1 / 2}, \\
& s_{y}=\left(\frac{1}{m} \sum_{i=1}^{m}\left(y_{i}-\bar{y}\right)^{2}\right)^{1 / 2},
\end{aligned}
$$

and

$$
s_{x y}=\frac{1}{m} \sum_{i=1}^{m}\left(x_{i}-\bar{x}\right)\left(y_{i}-\bar{y}\right),
$$

where $m$ is the number of the data sets used for the calculation and $\bar{x}(\bar{y})$ is the average value of $x(y)$. Then, the population for the next generation is constructed by inheriting the few elite functions with especially larger $|r|$ at the previous generation, ranking all the functions according to $|r|$, and eliminating a few inferior functions with especially higher $|r|$ from the population.

For a number of high-quality functions obtained by several GP searches, the predictive ability of the function is checked by a $k$-fold cross validation [14]. The data sets are randomly divided into $k$ subsets and a single subset is retained as the validation data for testing the functions and the remaining $k-1$ subsets are used for training the functions. In this study, we used $R=\left|r_{t s}\right|$ for the evaluation of the predictive ability, where $r_{t s}$ represents the correlation coefficient calculated from the testing data and takes the value from 0 to 1 . If the function developed by GP gives the strong correlation for the testing data, then $R$ shows a large value. This process is repeated $k$ times by rotation and the values of $R$ are averaged $(\bar{R})$. The function with the largest $\bar{R}$ is adopted as the predictor of the physical property which we will investigate.

\section{B. GA for crystal structure search}

Figure 5 shows a flowchart at each generation with respect to the structure search by GA. For the evolutionary operators, the mating is the operator to create a slab structure from two randomly selected individuals [Fig. 6(a)] and the mutation is the operator to give a lattice distortion or an atomic

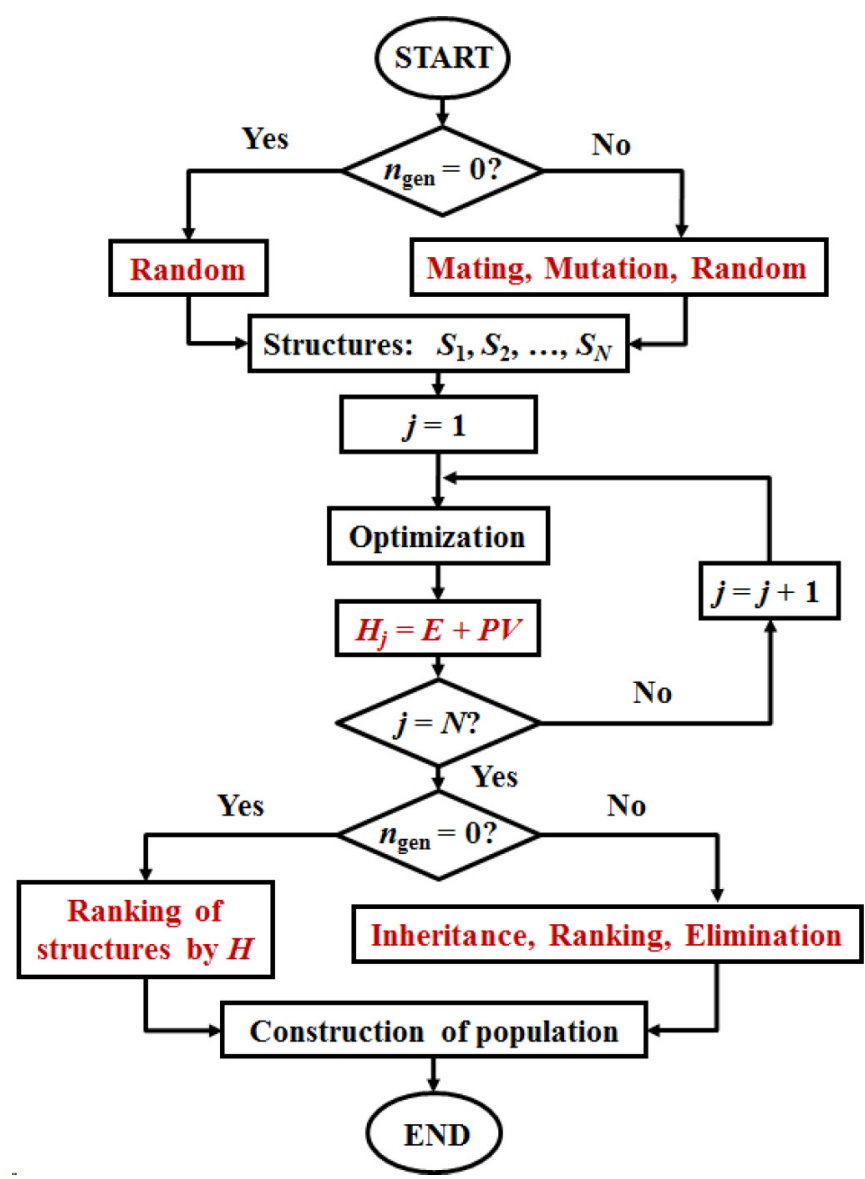

FIG. 5. Flowchart at each generation with respect to crystal structure prediction by GA.

permutation for a randomly selected individual [Fig. 6(b)] [11]. All the structures generated randomly or created by the mating and mutation are optimized at a given pressure, and the enthalpies, i.e., $H=E+P V$, are calculated using total energy $E$, pressure $P$, and volume $V$. Then, similar to the case of the predictor development, a high-quality crystal structure with low $H$ is developed by repeatedly performing the evolution, i.e., inheriting, ranking according to $H$, and eliminating.

(a)

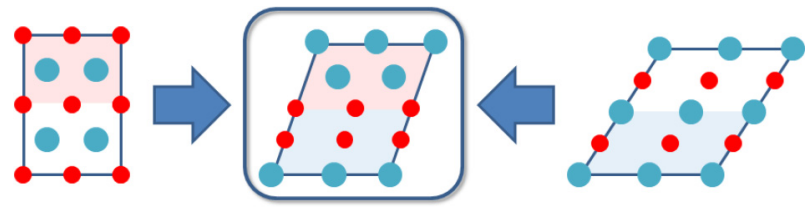

(b)
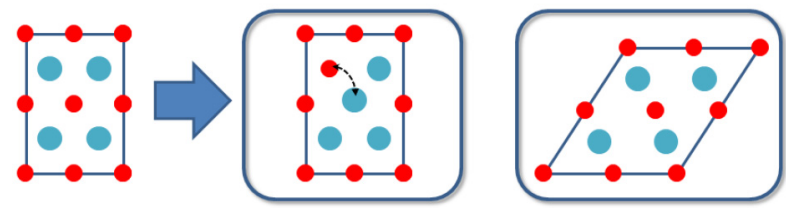

FIG. 6. Schematic view of crystal structure search by the evolutionary operations: (a) mating and (b) mutation. 

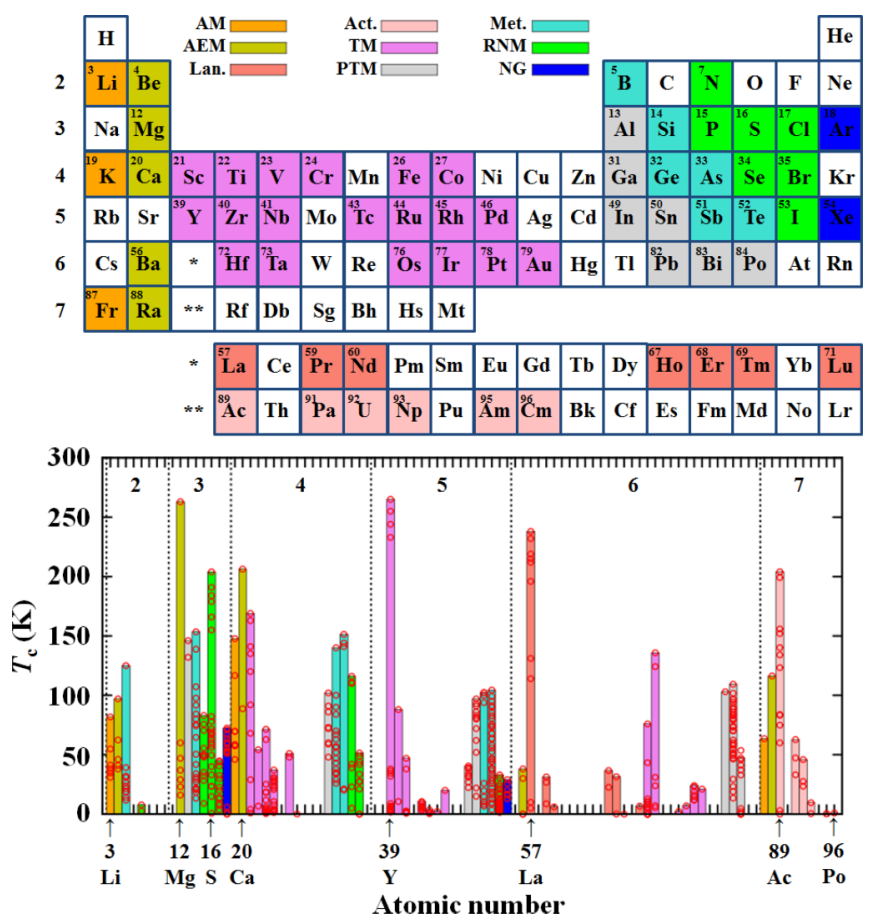

FIG. 7. Elements forming the binary hydrogen compounds included in the database and all the $T_{\mathrm{c}}$ data. The elements are divided into nine groups using different colors: Alkali metal (AM), alkaline earth metal (AEM), lanthanide (Lan.), actinide (Act.), transition metal (TM), post-transition metal (PTM), metalloid (Met.), reactive nonmetal (RNM), and noble gas (NG).

\section{APPLICATION TO SEARCH FOR SUPERCONDUCTING HYDROGEN COMPOUNDS}

We developed the calculation codes for the above GA and GP searches and applied our MI method to search for superconducting hydrogen compounds. In 2015, high-temperature superconductivity was discovered in hydrogen sulfide $\left(\mathrm{H}_{2} \mathrm{~S}\right)$ under high pressure and the superconducting critical temperature $T_{\mathrm{c}}$ reaches $203 \mathrm{~K}$ at a pressure of $155 \mathrm{GPa}$ [15]. In 2018-2019, compressed lanthanum hydrides broke the record and the superconductivity was observed at $250 \mathrm{~K}$ at $170 \mathrm{GPa}$ by Drozdov et al. [16] and $260 \mathrm{~K}$ at $190 \mathrm{GPa}$ by Somayazulu et al. [17]. Therefore, other hydrogen compounds also have a potential to become similar high- $T_{\mathrm{c}}$ superconductors under some conditions. However, there are a huge number of combinations for hydrogen compounds in multicomponent systems. For example, there exist 13572 combinations for ternary hydrogen compounds formed by all elements with the atomic numbers from 2 to 118 , and the number is much further increased by taking stoichiometry and crystal structure into account. Therefore, the MI approach is of great help to discover potential candidates with the high- $T_{\mathrm{c}}$ superconductivity.

\section{A. Data collection}

First we developed the database by collecting the firstprinciples calculation data with respect to chemical composition, crystal structure, and superconducting property of binary hydrogen compounds under high pressure condition from lit-
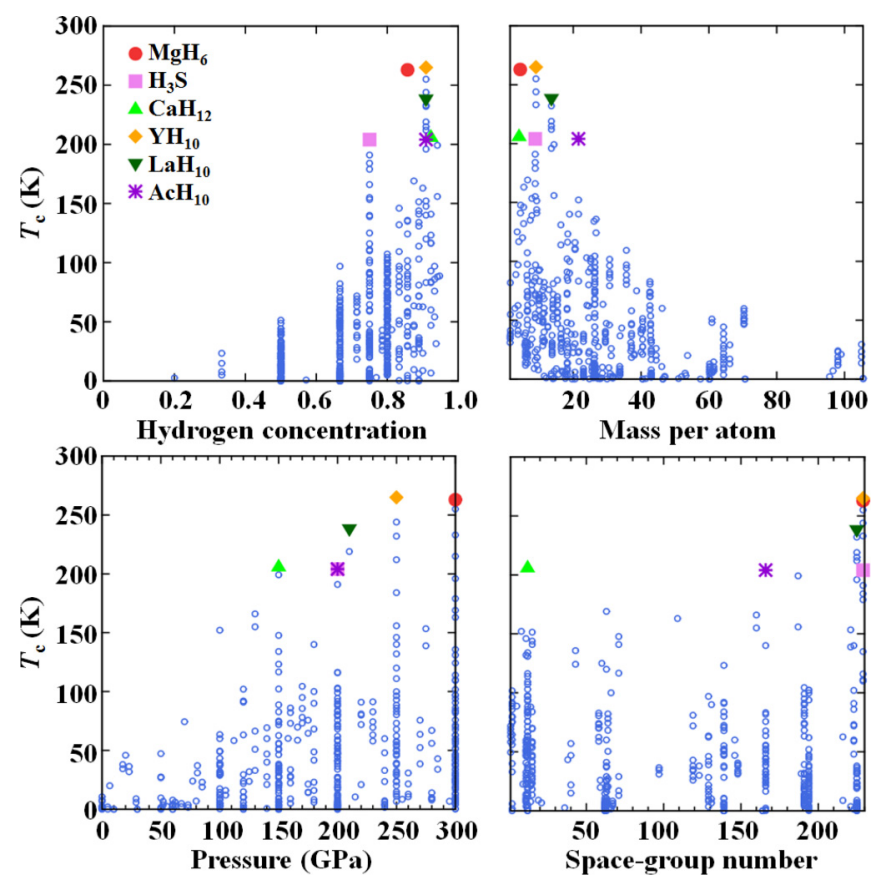

FIG. 8. Relationships between the superconducting $T_{\mathrm{c}}$ and hydrogen concentration (top left), $T_{\mathrm{c}}$ and mass per atom (top right), $T_{\mathrm{c}}$ and pressure (bottom left), and $T_{\mathrm{c}}$ and space-group number (bottom right) for superconducting binary hydrogen compounds predicted by first-principles calculations.

erature [18-81], many of which have not been experimentally realized yet. The database includes 497 data sets for the compounds with 62 elements colored in the Periodic Table of Fig. 7 . We used only the $T_{\mathrm{c}}$ data obtained by the Allen-Dynes formula [82] in this study, which are all plotted in the lower panel of Fig. 7. The highest $T_{\mathrm{c}}$ value for each binary system is represented as a histogram. The superconductivity of $T_{\mathrm{c}} \geqslant$ $200 \mathrm{~K}$ is predicted in the following compounds: $T_{\mathrm{c}}=265 \mathrm{~K}$ at $250 \mathrm{GPa}$ in $\mathrm{YH}_{10}$ [69], $263 \mathrm{~K}$ at $300 \mathrm{GPa}$ in $\mathrm{MgH}_{6}$ [50], $238 \mathrm{~K}$ at $210 \mathrm{GPa}$ in $\mathrm{LaH}_{10}$ [69], $206 \mathrm{~K}$ at $150 \mathrm{GPa}$ in $\mathrm{CaH}_{12}$ [77], $204 \mathrm{~K}$ at $200 \mathrm{GPa}$ in $\mathrm{AcH}_{10}$ [78], and $204 \mathrm{~K}$ at $200 \mathrm{GPa}$ in $\mathrm{H}_{3} \mathrm{~S}$ [34].

Figure 8 shows the relationships between $T_{\mathrm{c}}$ and hydrogen concentration, mass per atom, pressure, and space group number for all the data included in the database. Although large hydrogen concentration, light mass, and high pressure favor the increase of the superconductivity, they are not a necessary and sufficient condition for high- $T_{\mathrm{c}}$ superconductivity. Further, space group number, i.e., crystal symmetry, shows a weak correlation with $T_{\mathrm{c}}$. The data suggests that the search for hydrogen compounds with high $T_{\mathrm{c}}$ is seemingly simple, but it is actually difficult just to investigate these parameters independently.

\section{B. GP training}

Next we developed the superconductivity predictor by GP using the data sets included in the database. The data sets were divided into ten subsets for the cross-validation, i.e., $k=10$, and $90 \%$ of the data for training and $10 \%$ for testing. We used five variables, space group $(S)$, hydrogen concentration $(h)$, 


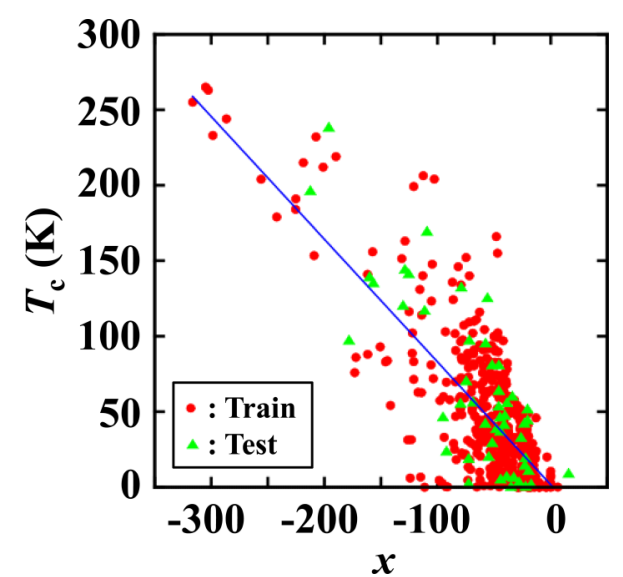

FIG. 9. Correlation between $T_{\mathrm{c}}$ and the superconductivity evaluation value $x$ calculated by the predictor. The training and testing data are represented as circle and triangle, respectively.

mass per atom $(M)$, pressure $(P)$, and the effective screened Coulomb repulsion constant in the Allen-Dynes formula [82] $\left(\mu^{*}\right)$, to obtain the superconductivity evaluation value $x ; x=$ $f\left(S, h, M, P, \mu^{*}\right)$ (see Fig. 3). The maximum length of a function, which is defined as the maximum value for the sum of the number of arithmetic operators, constants, and variables included in a function, was set at 500 in this study, whereas no constraint was used for the minimum length. The functions are evolved for 5000 generations, based on the correlation to the superconducting $T_{\mathrm{c}}(T) ; y=T$. The number of the individuals were set at 20, in which eight functions are created by the mating, eight by the mutation, two are randomly generated, and two are inherited. Then the 10-fold cross-validation was carried out and the $\bar{R}$ value was calculated. We performed this process 50 times in parallel and adopted the function with the largest $\bar{R}$ as the superconductivity predictor. Figure 9 shows the correlation between $x$ and $y\left(T_{\mathrm{c}}\right)$ obtained by the predictor. $\left|r_{t r}\right|$ and $\left|r_{t s}\right|$ are 0.78 and 0.81 , respectively, where $r_{t r}$ represents the correlation coefficient calculated from the training data and takes the value from 0 to 1 . The compounds taking small $x$ values are potential candidates for the high- $T_{\mathrm{c}}$ superconductivity. See Ref. [83] with respect to the details of the predictor, in which the function is represented as the Polish notation. The predictor is very complicated and uninterpretable because the function is heuristically created over many generations by GP. We consider that the interpretability can be increased by decreasing the maximum length of the functions in the GP search.

\section{GP prediction}

We searched for the potential candidates in ternary hydrogen compounds using this predictor. First, we created 497 data sets for ternary hydrogen compounds using all the data sets for the binary ones included in our database. That is to say, if in a binary compound the element paired with hydrogen has the atomic number of $Z$, then the corresponding ternary compound is created by replacing it with the elements having $Z-1$ and $Z+1$ as follows: $\mathrm{H}_{6} \mathrm{P}(Z=15) \mathrm{Cl}(Z=17)$ for $\mathrm{H}_{3} \mathrm{~S}(Z=16), \mathrm{Ba}(Z=56) \mathrm{Ce}(Z=58) \mathrm{H}_{20}$ for $\mathrm{La}(Z=57) \mathrm{H}_{10}$,
TABLE I. Superconductivity of the hypothetical ternary hydrogen compounds. The $T_{\mathrm{c}}$ values of the ternary compounds are estimated from the amount of change in the superconductivity evaluation value $x$.

\begin{tabular}{lcc}
\hline \hline Compounds & $x$ & $T_{\mathrm{c}}(\mathrm{K})$ \\
$\left(S, h, P, \mu^{*}\right)$ & $(M)$ & \\
\hline (a) Group 1-3 & & \\
$\mathrm{YH}_{10} \rightarrow \mathrm{SrZrH}_{20}$ & $-304.9 \rightarrow-292.7$ & $265[69] \rightarrow 255$ \\
$(229,0.9091,250,0.10)$ & $(9.00 \rightarrow 9.05)$ & \\
$\mathrm{MgH}_{6} \rightarrow \mathrm{NaAlH}_{12}$ & $-302.6 \rightarrow-287.5$ & $263[50] \rightarrow 251$ \\
$(229,0.8571,300,0.12)$ & $(4.34 \rightarrow 4.43)$ & \\
$\mathrm{LaH}_{10} \rightarrow \mathrm{BaCeH}_{20}$ & $-196.1 \rightarrow-196.4$ & $238[69] \rightarrow 238$ \\
$(225,0.9091,210,0.10)$ & $(13.54 \rightarrow 13.53)$ & \\
$\mathrm{CaH}_{12} \rightarrow \mathrm{KScH}_{24}$ & $-112.6 \rightarrow-113.7$ & $206[77] \rightarrow 207$ \\
$(12,0.9231,150,0.10)$ & $(4.01 \rightarrow 4.16)$ & \\
$\mathrm{AcH}_{10} \rightarrow \mathrm{RaThH}_{20}$ & $-102.9 \rightarrow-102.6$ & $204[78] \rightarrow 204$ \\
$(166,0.9091,200,0.10)$ & $(21.55 \rightarrow 21.73)$ & \\
$(b) \mathrm{Group} 13-16$ & & \\
$\mathrm{H}_{3} \mathrm{~S} \rightarrow \mathrm{H}_{6} \mathrm{PCl}$ & $-255.9 \rightarrow-152.2$ & $204[34] \rightarrow 120$ \\
$(229,0.7500,200,0.10)$ & $(8.77 \rightarrow 9.06)$ & \\
$\mathrm{SiH}_{3} \rightarrow \mathrm{AlPH}_{6}$ & $-209.1 \rightarrow-50.2$ & $153[21] \rightarrow 24$ \\
$(221,0.7500,275,0.10)$ & $(7.78 \rightarrow 8.00)$ & \\
$\mathrm{AsH}_{8} \rightarrow \mathrm{GeSeH}_{16}$ & $-131.6 \rightarrow-131.6$ & $151[65] \rightarrow 151$ \\
$(15,0.8889,450,0.10)$ & $(9.22 \rightarrow 9.32)$ & \\
$\mathrm{AlH}_{5} \rightarrow \mathrm{MgSiH}_{10}$ & $-81.7 \rightarrow-81.6$ & $146[47] \rightarrow 146$ \\
$(11,0.8333,250,0.10)$ & $(5.34 \rightarrow 5.21)$ & \\
$\mathrm{GeH}_{3} \rightarrow \mathrm{GaAsH}_{6}$ & $-71.9 \rightarrow-72.1$ & $140[33] \rightarrow 140$ \\
$(223,0.7500,180,0.13)$ & $(18.91 \rightarrow 18.84)$ & \\
\hline \hline
\end{tabular}

etc. The values of $S, h$, and $P$ were matched to those of the corresponding binary data. Moreover, we assumed that $\mu^{*}$ is less affected by the creation of the ternary compounds from the parent binary ones. In other words, only $M$ were varied with respect to the creation of the ternary data. Then, we calculated the $x$ values of the hypothetical ternary compounds using the created data sets. Table I lists a part of the results on $x$ and $T_{\mathrm{c}}$ for the hypothetical ternary compounds. We estimated the $T_{\mathrm{c}}$ values of the ternary compounds $\left(T_{\mathrm{c}}^{\mathrm{t}}\right)$ using the relationship of $T_{\mathrm{c}}^{\mathrm{t}}=-0.8130\left(x^{\mathrm{t}}-x^{\mathrm{b}}\right)+T_{\mathrm{c}}^{\mathrm{b}}$, where $x^{\mathrm{t}}\left(x^{\mathrm{b}}\right)$ is the evaluation value of the ternary (binary) compound and $T_{\mathrm{c}}^{\mathrm{b}}$ is the $T_{\mathrm{c}}$ value of the binary compounds obtained by the Allen-Dynes formula [82], included in the database. The slope of -0.8130 is taken from a simple linear regression model, $y=-0.8130 x+1.66$, which is obtained by a least-squares fitting of the data (see the line in Fig. 9). In the table, the results are classified into (a) the group 1-3 elements and (b) the group 13-16 elements. For the group 1-3 elements, in which polyhydrides with hydrogen cages are formed and $T_{\mathrm{c}}$ exceeds $200 \mathrm{~K}$, the superconductivity is slightly suppressed for $Y H_{10}$ and $\mathrm{MgH}_{6}$ and is less affected for $\mathrm{LaH}_{10}$ and $\mathrm{AcH}_{10}$ by changing to the hypothetical ternary compounds. Only $\mathrm{CaH}_{12}$ shows a slight enhancement of the superconductivity. For the group 13-16 elements, in which covalent hydrides are formed and $T_{\mathrm{c}}$ shows the values from 140 to $200 \mathrm{~K}$, the superconductivity is largely suppressed for $\mathrm{H}_{3} \mathrm{~S}$ and $\mathrm{SiH}_{3}$, whereas it is less affected for $\mathrm{AsH}_{8}, \mathrm{AlH}_{5}$, and $\mathrm{GeH}_{3}$. 

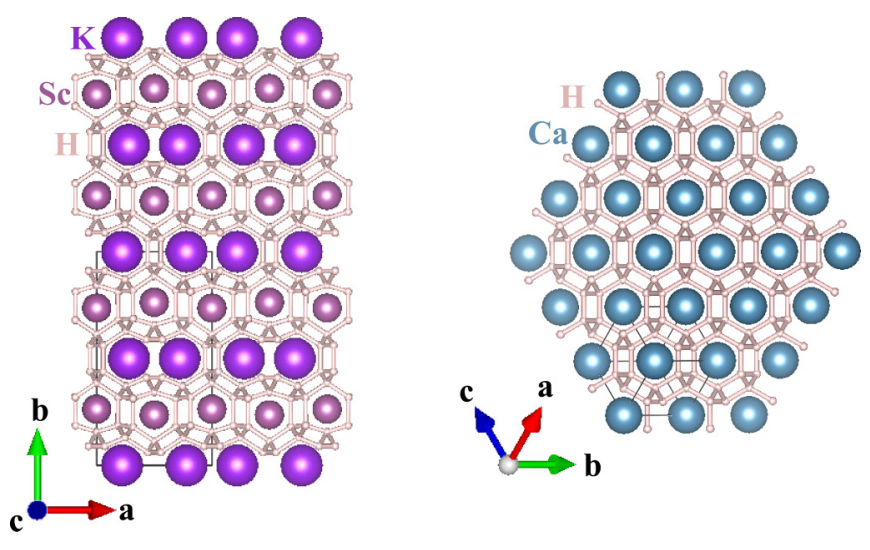

FIG. 10. (Left) Crystal structure of $\mathrm{KScH}_{12}$, predicted by GA. The space group is a monoclinic $C 2 / m$ (No. 12) with $a=$ 5.3726 ̊, $b / a=1.7795, c / a=0.5131, \beta=106.6^{\circ}, \mathrm{K}: 4 i(0.77998$, $0,0.47704)$, Sc: $4 g(0,0.26336,0), \mathrm{H} 1: 8 j(0.74515,0.37079$, 0.01997), H2: $8 j$ (-0.05716, 0.43888, 0.14288), H3: $8 j$ (0.57041, $0.41013,-0.05075), \mathrm{H} 4: 8 j$ (0.81603, 0.18680, 0.40624), H5: $8 j$ (0.68641, 0.18066, 0.59388), H6: $4 h(0,0.38665,0.5)$, and H7: $4 h$ $(0,0.84338,0.5)$ at $300 \mathrm{GPa}$. (Right) Crystal structure of $I m-3 m \mathrm{CaH}_{6}$ reported earlier [84]. Crystal structures were drawn with VESTA [88].

\section{GA structure search and first-principles validation}

\section{1. $\mathrm{KScH}_{12}$}

We verified the superconductivity of one of the potential candidates, $\mathrm{KScH}_{12}$, which is a hypothetical ternary compound approximated to $\mathrm{CaH}_{6}$ and is included in the group 1-3 elements. $\mathrm{CaH}_{6}$ is predicted to form the hydrogen cage structure under high pressure and show $T_{\mathrm{c}}$ of $220-235 \mathrm{~K}$ at $150 \mathrm{GPa}$, which is obtained by numerically solving the Eliashberg equations [84]. Therefore, $\mathrm{KScH}_{12}$ is expected to show similar crystal structure and superconductivity under high pressure.

The variation of $T_{\mathrm{c}}$ shown in Table $\mathrm{I}$ is estimated under the hypothesis that the space group $S$ is invariant between binary and ternary compounds. However, $S$ for the most stable structure of the ternary compound is considered to be different from that of the binary one. Therefore, first we searched for the most stable structure of $\mathrm{KScH}_{12}$ by combining our GA code with the QUANTUM ESPRESSO (QE) code [85] and applying it to $\mathrm{KScH}_{12}$. In our GA search, eight structures are created by "mating," six "mutation (distortion)," and six "mutation (permutation)" in each generation. We performed the structure search at pressures of 200 and $300 \mathrm{GPa}$, using calculation cells including 1-4 formula units. The generalized gradient approximation by Perdew, Burke, and Ernzerhof [86] was used for the exchange-correlation functional, and the Rabe-Rappe-Kaxiras-Joannopoulos ultrasoft pseudopotential [87] was employed. The $k$-space integration over the Brillouin zone (BZ) was carried out on a $4 \times 4 \times 4$ grid, and the energy cutoff was set at $80 \mathrm{Ry}$ for the wave function and $640 \mathrm{Ry}$ for the charge density. After we obtained the stable structures, we improved the calculation accuracy by increasing the number of $k$ points and checked the stability. As the results, we obtained a monoclinic $C 2 / m$ (No. 12) structure at $300 \mathrm{GPa}$, where it shows no phonon instability. The structure takes a modulated hydrogen-cage structure, similar to the structure
TABLE II. Variation of superconductivity evaluation value $x$ and superconducting $T_{\mathrm{c}}\left(\Delta T_{\mathrm{c}}\right)$ due to the change from $\mathrm{Im}-3 m \mathrm{CaH}_{6}$ to $C 2 / m \mathrm{KScH}_{12}$ at $300 \mathrm{GPa}$.

\begin{tabular}{lcc}
\hline \hline $\mathrm{CaH}_{6} \rightarrow \mathrm{KScH}_{12}$ & $x$ & $\Delta T_{\mathrm{c}}(\mathrm{K})$ \\
\hline$I m-3 m \rightarrow C 2 / m$ & $-179.5 \rightarrow-95.5$ & -68 \\
\hline \hline
\end{tabular}

of $\mathrm{CaH}_{6}$ (compare the structure between the left and right in Fig. 10). The evaluation values of $I m-3 m \mathrm{CaH}_{6}(S=229)$ and $C 2 / \mathrm{mKScH}_{12}(S=12)$ are calculated to be -179.5 and -95.5 at $300 \mathrm{GPa}$, respectively (Table II). That is to say, $T_{\mathrm{c}}$ decreases by about $68 \mathrm{~K}$ owing to the change from $\mathrm{Im}-3 m \mathrm{CaH}_{6}$ to $C 2 / m \mathrm{KScH}_{12}$ according to the slope of -0.8130 mentioned in Sec. III C.

Next, we investigated the superconductivity of $C 2 / m \mathrm{KScH}_{12}$ using first-principles calculations. $T_{\mathrm{c}}$ was calculated using the Allen-Dynes formula [82],

$$
T_{\mathrm{c}}=\frac{\omega_{\log }}{1.2} \exp \left[-\frac{1.04(1+\lambda)}{\lambda-\mu^{*}(1+0.62 \lambda)}\right] .
$$

In Eq. (4), the parameters of electron-phonon coupling constant $\lambda$ and logarithmic-averaged phonon frequency $\omega_{\log }$ represent a set of characters for the phonon-mediated superconductivity. To obtain these parameters, we performed the phonon calculations implemented in the QE code. We used an $8 \times 16 \times 8 k$-point grid for the electron-phonon calculation, and $4 \times 8 \times 4 k$-point and $4 \times 4 \times 4 q$-point grids for the dynamical matrix calculation. We also calculated $T_{\mathrm{c}}$ of Im- $3 m \mathrm{CaH}_{6}$ using a $24 \times 24 \times 24 k$-point grid, and $12 \times$ $12 \times 12 k$-point and $4 \times 4 \times 4 q$-point grids. The effective screened Coulomb repulsion constant $\mu^{*}$ was assumed to be $0.10-0.13$, which has been considered to be a reasonable value for hydrides. The results are listed in Table III. $T_{\mathrm{c}}$ of Im $-3 m \mathrm{CaH}_{6}$ at $150 \mathrm{GPa}$ is $172 \mathrm{~K}\left(\mu^{*}=0.13\right)$, which is lower by about $50 \mathrm{~K}$ than that obtained by the different method, i.e., the numerical solution of the Eliashberg equation, reported earlier [84]. Other hydrogen compounds also show the similar tendency that $T_{\mathrm{c}}$ is underestimated when the Allen-Dynes formula is used $[69,89] . T_{\mathrm{c}}$ of $C 2 / \mathrm{mKScH}_{12}$ shows $122 \mathrm{~K}$ $\left(\mu^{*}=0.13\right)$ at $300 \mathrm{GPa}$, which is decreased by $38 \mathrm{~K}$ owing to the change from $I m-3 m \mathrm{CaH}_{6}$. This result indicates that the

TABLE III. Superconductivity of $\mathrm{CaH}_{6}$ and $\mathrm{KScH}_{12}$, obtained by the first-principles calculations and the Allen-Dynes formula. $T_{\mathrm{c}}$ was calculated using $\mu^{*}=0.10$ and 0.13 . The values in the parentheses show the $T_{\mathrm{c}}$ data estimated from the regression line of $y=-0.8130 x+1.66$ using the evaluation value $x$.

\begin{tabular}{lcccccc}
\hline \hline & & & & \multicolumn{3}{c}{$T_{\mathrm{c}}(\mathrm{K})$} \\
& $S$ & $P(\mathrm{GPa})$ & $\lambda$ & $\omega_{\ln }$ & 0.10 & 0.13 \\
\hline $\mathrm{CaH}$ & $I m-3 m$ & 150 & 2.55 & 1091 & 182 & 172 \\
& & & & & $(128)$ & $(121)$ \\
& & 300 & 1.66 & 1388 & 174 & 160 \\
& & & & & $(155)$ & $(148)$ \\
$\mathrm{KScH}_{12}$ & $C 2 / m$ & 300 & 1.54 & 1139 & 133 & 122 \\
& & & & & $(81)$ & $(79)$ \\
\hline \hline
\end{tabular}



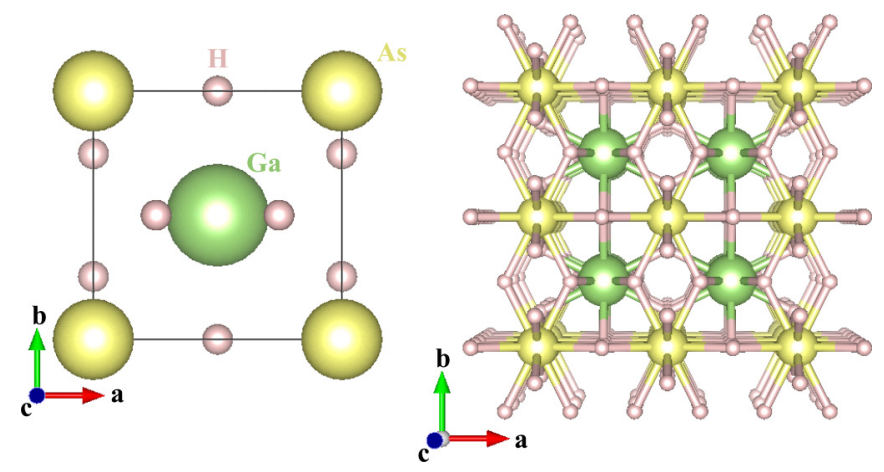

FIG. 11. Crystal structure of $\mathrm{GaAsH}_{6}$, predicted by GA. The space group is a cubic Pm-3 (No. 200) with $a=3.1050 \AA$, Ga: $1 b$ $(0.5,0.5,0.5)$, As: $1 a(0,0,0)$, and $\mathrm{H}: 6 g(0.74736,0.5,0)$. Crystal structure was drawn with VESTA [88].

decrease of $T_{\mathrm{c}}$ is qualitatively consistent with that estimated from the variation of the evaluation value $x$ shown in Table II.

\section{2. $\mathrm{GaAsH}_{6}$}

We verified the superconductivity of another candidate, $\mathrm{GaAsH}_{6}$, included in the group 13-16 elements. Gallium arsenide (GaAs) has been well known as a III-V direct semiconductor at ambient pressure, and the hydrogenation of the compound is expected to be achieved under high-pressure conditions, as well as group IV semiconductors. We searched for stable structures at pressures of 50,100, 200, and $300 \mathrm{GPa}$, using calculation cells including 1-4 formula units. The $k$ space integration over the Brillouin zone (BZ) was performed on an $8 \times 8 \times 8$ grid, and the energy cutoff was set at 80 Ry for the wave function and 640 Ry for the charge density. As the results, we obtained a cubic Pm-3 (No. 200) structure at $180 \mathrm{GPa}$ (Fig. 11). Although the structure is similar to the A15 structure with $\mathrm{Pm}-3 n$ (No. 223) of $\mathrm{GeH}_{3}$ reported earlier [33], the space group is different from that of the A15 structure. The evaluation value $x$ of $P m-3 \mathrm{GaAsH}_{6}$ is calculated to be -40.0 at $180 \mathrm{GPa}$, which gives the $T_{\mathrm{c}}$ variations of $-8 \mathrm{~K}$ from Cccm $\mathrm{GeH}_{3},-7 \mathrm{~K}$ from $P 4_{2} / m m c \mathrm{GeH}_{3}$, and $-26 \mathrm{~K}$ from $P m-3 n \mathrm{GeH}_{3}$ (Table IV). That is to say, $T_{\mathrm{c}}$ is slightly varied according to the change from $C c c m$ or $P 4_{2} / \mathrm{mmcGeH}_{3}$ to $P m-3 \mathrm{GaAsH}_{6}$, whereas it is more largely decreased according to the change from $\mathrm{Pm}-3 n \mathrm{GeH}_{3}$.

We investigated the superconductivity of $\mathrm{Pm}-3 \mathrm{GaAsH}_{6}$ using first-principles calculations and the Allen-Dynes formula. We used a $48 \times 48 \times 48 k$-point grid for the electronphonon calculation, and $24 \times 24 \times 24 k$-point and $6 \times 6 \times 6$

TABLE IV. Variation of superconductivity evaluation value $x$ and superconducting $T_{\mathrm{c}}\left(\Delta T_{\mathrm{c}}\right)$ due to the change from $\mathrm{GeH}_{3}$ to $\mathrm{GaAsH}_{6}$ at $180 \mathrm{GPa}$. The three data sets of $\mathrm{GeH}_{3}, P m-3 n$ (No. 223) $\mathrm{GeH}_{3}, C c c m$ (No. 66) $\mathrm{GeH}_{3}$, and $P 4_{2} / m m c$ (No. 131) are taken from Ref. [33].

\begin{tabular}{lcc}
\hline \hline $\mathrm{GeH}_{3} \rightarrow \mathrm{GaAsH}_{6}$ & $x$ & $\Delta T_{\mathrm{c}}(\mathrm{K})$ \\
\hline $\mathrm{Cccm} \rightarrow \mathrm{Pm}-3$ & $-49.7 \rightarrow-40.0$ & -8 \\
$P 4_{2} / m m c \rightarrow P m-3$ & $-48.8 \rightarrow-40.0$ & -7 \\
$P m-3 n \rightarrow P m-3$ & $-71.9 \rightarrow-40.0$ & -26 \\
\hline \hline
\end{tabular}

TABLE V. Superconductivity of $\mathrm{GeH}_{3}$ and $\mathrm{GaAsH}_{6}$ obtained by the first-principles calculations and the Allen-Dynes formula. $T_{\mathrm{c}}$ was calculated using $\mu^{*}=0.10$ and 0.13 . The data of $\mathrm{GeH}_{3}$ is taken from Ref. [33]. The values in the parentheses show the $T_{\mathrm{c}}$ data estimated from the regression line of $y=-0.8130 x+1.66$ using the evaluation value $x$.

\begin{tabular}{|c|c|c|c|c|c|c|}
\hline & \multirow[b]{2}{*}{$S$} & \multirow[b]{2}{*}{$P(\mathrm{GPa})$} & \multirow[b]{2}{*}{$\lambda$} & \multirow[b]{2}{*}{$\omega_{\ln }$} & \multicolumn{2}{|c|}{$T_{\mathrm{c}}(\mathrm{K})$} \\
\hline & & & & & 0.10 & 0.13 \\
\hline \multirow[t]{3}{*}{$\mathrm{GeH}_{3}$} & Cccm & 180 & 1.60 & 793 & $\begin{array}{l}110 \\
(42)\end{array}$ & $\begin{array}{l}100 \\
(42)\end{array}$ \\
\hline & $P 4_{2} / m m c$ & & 1.56 & 737 & $\begin{array}{l}100 \\
(42)\end{array}$ & $\begin{array}{c}90 \\
(41)\end{array}$ \\
\hline & $P m-3 n$ & & 1.82 & 989 & $\begin{array}{l}160 \\
(61)\end{array}$ & $\begin{array}{l}140 \\
(60)\end{array}$ \\
\hline \multirow[t]{3}{*}{$\mathrm{GaAsH}_{6}$} & $P m-3$ & 180 & 1.57 & 897 & $\begin{array}{l}107 \\
(35)\end{array}$ & $\begin{array}{c}98 \\
(34)\end{array}$ \\
\hline & & 200 & 1.43 & 964 & $\begin{array}{l}105 \\
(35)\end{array}$ & $\begin{array}{c}96 \\
(35)\end{array}$ \\
\hline & & 300 & 1.57 & 635 & $\begin{array}{c}76 \\
(38)\end{array}$ & $\begin{array}{c}69 \\
(37)\end{array}$ \\
\hline
\end{tabular}

$q$-point grids for the dynamical matrix calculation. The effective screened Coulomb repulsion constant $\mu^{*}$ was assumed to be $0.10-0.13$. The results are listed in Table V. $T_{\mathrm{c}}$ of $P m-3$ $\mathrm{GaAsH}_{6}$ shows $98 \mathrm{~K}\left(\mu^{*}=0.13\right)$ at $180 \mathrm{GPa}$, which is lower by $42 \mathrm{~K}$ than that of a metastable $P m-3 n$ phase of $\mathrm{GeH}_{3}\left(T_{\mathrm{c}}=\right.$ $140 \mathrm{~K}$ ) but is comparable with that of the most stable $\mathrm{Cccm}$ phase $\left(T_{\mathrm{c}}=100 \mathrm{~K}\right)$. Thus the $T_{\mathrm{c}}$ variations are qualitatively consistent with those estimated from the evaluation value $x$.

The values in the parentheses in Tables III and V show the $T_{\mathrm{c}}$ data estimated from the regression line of $y=-0.8130 x+$ 1.66 using the evaluation value $x$. Although the values are not consistent with the $T_{\mathrm{c}}$ data calculated by the Allen-Dynes formula owing to the errors from the regression line, which is more increased in low $T_{\mathrm{c}}$ region, the superconductivity can be predicted approximately. These results suggest that the regression analysis based on the superconductivity predictor gives reliable results on potential candidates for the high- $T_{\mathrm{c}}$ superconductivity in the ternary hydrogen compounds approximated to binary ones included in the database.

\section{DISCUSSION AND CONCLUSION}

We developed the MI method consisting of the five stages, which is based on EA: (i) data collection, (ii) GP training, (iii) GP prediction, (iv) GA structure search, and (v) first-principles validation. Turning of the cycle enables one to accelerate the search for novel functional materials. We applied the MI method to search for the superconducting hydrogen compounds. First, we developed the database on the superconductivity of the binary hydrogen compounds by collecting the first-principles data from literature, many of which have not been experimentally realized yet. Then, we developed the superconductivity predictor and explored the superconductivity in the hypothetical ternary hydrogen compounds, based on the binary compounds in the database. For the group 1-3 elements, the superconductivity is less affected by changing to the ternary compounds, and 
high- $T_{\mathrm{c}}$ superconductivity is expected in the hypothetical ternary compounds, as is the case with the binary ones. For the group 13-16 elements, the superconductivity is largely suppressed in the ternary compounds based on $\mathrm{H}_{3} \mathrm{~S}$ and $\mathrm{SiH}_{3}$, whereas those based on $\mathrm{AsH}_{8}, \mathrm{AlH}_{5}$, and $\mathrm{GeH}_{3}$ are less affected by the changing. We actually verified the superconductivity of $\mathrm{KScH}_{12}$ created from $\mathrm{CaH}_{6}$ and $\mathrm{GaAsH}_{6}$ created from $\mathrm{GeH}_{3}$ using the first-principles calculations. For $\mathrm{KScH}_{12}$, a modulated hydrogen-cage structure with a space group of $C 2 / m$ is obtained at $300 \mathrm{GPa}$ by GA, and $T_{\mathrm{c}}$ shows $122 \mathrm{~K}$ $\left(\mu^{*}=0.13\right)$. For $\mathrm{GaAsH}_{6}$, we predicted a cubic $\mathrm{Pm}-3$ structure and obtained $T_{\mathrm{c}}$ of $98 \mathrm{~K}\left(\mu^{*}=0.13\right)$ at $180 \mathrm{GPa}$. These $T_{\mathrm{c}}$ values are qualitatively consistent with those estimated from the variations of the superconductivity evaluation values. These results suggest that the regression analysis based on the superconductivity predictor is effective for the discovery of potential candidates in the ternary hydrogen compounds.

Although the superconductivity of only two hypothetical ternary compounds, $\mathrm{KScH}_{12}$ and $\mathrm{GaAsH}_{6}$, was validated by first-principles calculations, other compounds listed in Table I also have a potential to show high- $T_{\mathrm{c}}$ superconductivity and further validations are required. We have not investigated the stability of $\mathrm{KScH}_{12}$ and $\mathrm{GaAsH}_{6}$ with respect to the dissociation yet. However, their parent binary compounds, $\mathrm{CaH}_{6}$ and $\mathrm{GeH}_{3}$, are predicted to be thermodynamically stable phases, and the ternary compounds are expected to be stable or acceptably metastable. Very recently, Sun et al. predicted the remarkably high- $T_{\mathrm{c}}$ superconductivity, $473 \mathrm{~K}$ at $250 \mathrm{GPa}$, in metastable ternary $\mathrm{Li}_{2} \mathrm{MgH}_{16}$ [90]. By aggregating the superconductivity data of $\mathrm{KScH}_{12}, \mathrm{GaAsH}_{6}, \mathrm{Li}_{2} \mathrm{MgH}_{16}$, and other ternary compounds into the database and redeveloping the predictor, the prediction accuracy for the superconductivity in ternary hydrogen compounds is further increased. At present, the superconductivity of $T_{\mathrm{c}} \geqslant 200 \mathrm{~K}$ is found only in the pressure above $150 \mathrm{GPa}$. Therefore, the achievement of $T_{\mathrm{c}}$ higher than $200 \mathrm{~K}$ at low pressure is also significantly important, and MI approaches could be effective for the research.

In our GP search, we used the arithmetic operators $(+,-, \times, \div)$, the constants $(1-10)$, and the five variables $\left(S, h, M, P, \mu^{*}\right)$ to create the functions. The data of the five variables is easily collected from theoretical papers reported earlier, in spite of a small amount of physical and chemical property data with respect to hydrogen compounds under high pressure. Even under such a constraint, accurate predictors can be obtained by the combination of these operators, constants, and variables because the functions are heuristically created over many generations by GP. We expect that the prediction accuracy will be further increased by adding other physical and chemical property parameters into the variables and adding mathematical functions such as trigonometric functions, exponential function, etc. into the leaf nodes.

The purpose of this study is to construct a system for materials discovery within the framework of the evolutionary algorithm and, therefore, we adopted the GP technique for the development of the predictor. As another machine learning technique, an artificial neural network (ANN) has been known as a powerful tool to develop the predictor for big data, whereas the interpretation of the predictor is difficult owing to its black box nature. GP is a simple and effective approach to develop the interpretable predictor for the case of the small number of data sets and parameters like this study (note that the interpretability can be increased by decreasing the maximum length of the functions). Although the prediction accuracy may be decreased compared with ANN, it is compensated by performing the first-principles validation and turning the MI cycle shown in Fig. 1. There are other techniques such as decision trees, support vector machines, Bayesian networks, kernel ridge regression, etc. with respect to the machine learning. The MI method can be further improved by combining these techniques.

\section{ACKNOWLEDGMENTS}

This work was supported by JSPS KAKENHI under Grant-in-Aid for Specially Promoted Research (Grant No. 26000006), Scientific Research (C) (Grant No. 17K05541) and Scientific Research (S) (Grant No. 16H06345), MEXT under ESICMM (Grant No. 12016013) and "Exploratory Challenge on Post-K computerh (Frontiers of Basic Science: Challenging the Limits)," Asahi Glass Foundation, and Yamada Science Foundation.
[1] R. Potyrailo, K. Rajan, K. Stoewe, I. Takeuchi, and B. Chisholm, ACS Comb. Sci. 13, 579 (2011).

[2] A. Jain, S. P. Ong, G. Hautier, W. Chen, W. D. Richards, S. Dacek, S. Cholia, D. Gunter, D. Skinner, G. Ceder et al., APL Materials 1, 011002 (2013).

[3] S. P. Ong, W. D. Richards, A. Jain, G. Hautier, M. Kocher, S. Cholia, D. Gunter, V. L. Chevrier, K. A. Persson, and G. Ceder, Comput. Mater. Sci. 68, 314 (2013).

[4] K. Takahashi and Y. Tanaka, Dalton Trans. 45, 10497 (2016).

[5] M. Nishijima, T. Ootani, Y. Kamimura, T. Sueki, S. Esaki, S. Murai, K. Fujita, K. Tanaka, K. Ohira, Y. Koyama et al., Nat. Commun. 5, 4553 (2014).

[6] V. Stanev, C. Oses, A. G. Kusne, E. Rodriguez, J. Paglione, S. Curtarolo, and I. Takeuchi, Npj Comput. Mater. 4, 29 (2018)

[7] D. M. Deaven and K. M. Ho, Phys. Rev. Lett. 75, 288 (1995).
[8] T. S. Bush, C. R. A. Catlow, and P. D. Battle, J. Mater. Chem 5, 1269 (1995).

[9] S. M. Woodley, P. D. Battle, J. D. Gale, and C. R. A. Catlow, Phys. Chem. Chem. Phys. 1, 2535 (1999).

[10] S. M. Woodley, Struct. Bonding 110, 95 (2004).

[11] A. R. Oganov and C. W. Glass, J. Chem. Phys. 124, 244704 (2006).

[12] N. L. Abraham and M. I. J. Probert, Phys. Rev. B 73, 224104 (2006).

[13] N. L. Abraham and M. I. J. Probert, Phys. Rev. B 77, 134117 (2008).

[14] M. Stone, J. R. Stat. Soc. B 36, 111 (1974).

[15] A. P. Drozdov, M. I. Eremets, I. A. Troyan, V. Ksenofontov, and S. I. Shylin, Nature (London) 525, 73 (2015).

[16] A. P. Drozdov, P. P. Kong, V. S. Minkov, S. P. Besedin, M. A. Kuzovnikov, S. Mozaffari, L. Balicas, F. F. Balakirev, D. E. Graf, V. B. Prakapenka et al., Nature (London) 569, 528 (2019). 
[17] M. Somayazulu, M. Ahart, A. K. Mishra, Z. M. Geballe, M. Baldini, Y. Meng, V. V. Struzhkin, and R. J. Hemley, Phys. Rev. Lett. 122, 027001 (2019).

[18] X.-J. Chen, J.-L. Wang, V. V. Struzhkin, H. K. Mao, R. J. Hemley, and H.-Q. Lin, Phys. Rev. Lett. 101, 077002 (2008).

[19] D. Y. Kim, R. H. Scheicher, and R. Ahuja, Phys. Rev. Lett. 103, 077002 (2009).

[20] J. S. Tse, Z. Song, Y. Yao, J. S. Smith, S. Desgreniers, and D. D. Klug, Solid State Commun. 149, 1944 (2009).

[21] X. Jin, X. Meng, Z. He, Y. Ma, B. Liu, T. Cui, G. Zou, and H. k. Mao, Proc. Natl. Acad. Sci. USA 107, 9969 (2010).

[22] D. Duan, F. Tian, Z. He, X. Meng, L. Wang, C. Chen, X. Zhao, B. Liu, and T. Cui, J. Chem. Phys. 133, 074509 (2010).

[23] G. Gao, A. R. Oganov, P. Li, Z. Li, H. Wang, T. Cui, Y. Ma, A. Bergara, A. O. Lyakhov, T. Iitaka et al., Proc. Natl. Acad. Sci. USA 107, 1317 (2010).

[24] D. Y. Kim, R. H. Scheicher, C. J. Pickard, R. J. Needs, and R. Ahuja, Phys. Rev. Lett. 107, 117002 (2011).

[25] G. Gao, H. Wang, A. Bergara, Y. Li, G. Liu, and Y. Ma, Phys. Rev. B 84, 064118 (2011).

[26] K. Abe and N. W. Ashcroft, Phys. Rev. B 84, 104118 (2011).

[27] C. Zhang, X.-J. Chen, and H.-Q. Lin, J. Phys.: Condens. Matter 24, 035701 (2012).

[28] D. Zhou, X. Jin, X. Meng, G. Bao, Y. Ma, B. Liu, and T. Cui, Phys. Rev. B 86, 014118 (2012).

[29] D. C. Lonie, J. Hooper, B. Altintas, and E. Zurek, Phys. Rev. B 87, 054107 (2013).

[30] G. Gao, R. Hoffmann, N. W. Ashcroft, H. Liu, A. Bergara, and Y. Ma, Phys. Rev. B 88, 184104 (2013).

[31] C.-H. Hu, A. R. Oganov, Q. Zhu, G.-R. Qian, G. Frapper, A. O. Lyakhov, and H.-Y. Zhou, Phys. Rev. Lett. 110, 165504 (2013).

[32] J. Hooper, B. Altintas, A. Shamp, and E. Zurek, J. Phys. Chem. C 117, 2982 (2013).

[33] K. Abe and N. W. Ashcroft, Phys. Rev. B 88, 174110 (2013).

[34] D. Duan, Y. Liu, F. Tian, D. Li, X. Huang, Z. Zhao, H. Yu, B. Liu, W. Tian, and T. Cui, Sci. Rep. 4, 6968 (2014).

[35] C. Chen, F. Tian, D. Duan, K. Bao, X. Jin, B. Liu, and T. Cui, J. Chem. Phys. 140, 114703 (2014).

[36] I. Errea, M. Calandra, and F. Mauri, Phys. Rev. B 89, 064302 (2014).

[37] S. Yu, Q. Zeng, A. R. Oganov, C. Hu, G. Frapper, and L. Zhang, AIP Adv. 4, 107118 (2014).

[38] Y. Li, J. Hao, H. Liu, Y. Li, and Y. Ma, J. Chem. Phys. 140, 174712 (2014).

[39] Y. Xie, Q. Li, A. R. Oganov, and H. Wang, Acta Crystallogr., C 70, 104 (2014).

[40] Z. Wang, Y. Yao, L. Zhu, H. Liu, T. Iitaka, H. Wang, and Y. Ma, J. Chem. Phys. 140, 124707 (2014).

[41] C. Chen, Y. Xu, X. Sun, and S. Wang, J. Phys. Chem. C 119, 17039 (2015).

[42] D. Duan, F. Tian, Y. Liu, X. Huang, D. Li, H. Yu, Y. Ma, B. Liu, and T. Cui, Phys. Chem. Chem. Phys. 17, 32335 (2015).

[43] A. Shamp and E. Zurek, J. Phys. Chem. Lett. 6, 4067 (2015).

[44] H. Zhang, X. Jin, Y. Lv, Q. Zhuang, Q. Lv, Y. Liu, K. Bao, D. Li, B. Liu, and T. Cui, Phys. Chem. Chem. Phys. 17, 27630 (2015).

[45] H. Zhang, X. Jin, Y. Lv, Q. Zhuang, Y. Liu, Q. Lv, K. Bao, D. Li, B. Liu, and T. Cui, Sci. Rep. 5, 8845 (2015).
[46] I. Errea, M. Calandra, C. J. Pickard, J. Nelson, R. J. Needs, Y. Li, H. Liu, Y. Zhang, Y. Ma, and F. Mauri, Phys. Rev. Lett. 114, 157004 (2015).

[47] P. Hou, X. Zhao, F. Tian, D. Li, D. Duan, Z. Zhao, B. Chu, B. Liu, and T. Cui, RSC Adv. 5, 5096 (2015).

[48] S. Yu, X. Jia, G. Frapper, D. Li, A. R. Oganov, Q. Zeng, and L. Zhang, Sci. Rep. 5, 17764 (2015).

[49] S. Zhang, Y. Wang, J. Zhang, H. Liu, X. Zhong, H.-F. Song, G. Yang, L. Zhang, and Y. Ma, Sci. Rep. 5, 15433 (2015).

[50] X. Feng, J. Zhang, G. Gao, H. Liu, and H. Wang, RSC Adv. 5, 59292 (2015).

[51] X. Yan, Y. Chen, X. Kuang, and S. Xiang, J. Chem. Phys. 143, 124310 (2015).

[52] Y. Cheng, C. Zhang, T. Wang, G. Zhong, C. Yang, X.-J. Chen, and H.-Q. Lin, Sci. Rep. 5, 16475 (2015).

[53] Y. Liu, D. Duan, F. Tian, D. Li, X. Sha, Z. Zhao, H. Zhang, G. Wu, H. Yu, B. Liu et al., arXiv:1503.08587.

[54] Y. Liu, D. Duan, F. Tian, H. Liu, C. Wang, X. Huang, D. Li, Y. Ma, B. Liu, and T. Cui, Inorg. Chem. 54, 9924 (2015).

[55] Y. Liu, D. Duan, X. Huang, F. Tian, D. Li, X. Sha, C. Wang, H. Zhang, T. Yang, B. Liu et al., J. Phys. Chem. C 119, 15905 (2015).

[56] Y. Liu, X. Huang, D. Duan, F. Tian, H. Liu, D. Li, Z. Zhao, X. Sha, H. Yu, H. Zhang et al., Sci. Rep. 5, 11381 (2015).

[57] Y. Ma, D. Duan, D. Li, Y. Liu, F. Tian, H. Yu, C. Xu, Z. Shao, B. Liu, and T. Cui, arXiv:1511.05291.

[58] A. Shamp, T. Terpstra, T. Bi, Z. Falls, P. Avery, and E. Zurek, J. Am. Chem. Soc. 138, 1884 (2016).

[59] H. Liu, Y. Li, G. Gao, J. S. Tse, and I. I. Naumov, J. Phys. Chem. C 120, 3458 (2016).

[60] G.-R. Qian, H. Niu, C.-H. Hu, A. R. Oganov, Q. Zeng, and H.-Y. Zhou, Sci. Rep. 6, 25947 (2016).

[61] M. M. D. Esfahani, Z. Wang, A. R. Oganov, H. Dong, Q. Zhu, S. Wang, M. S. Rakitin, and X.-F. Zhou, Sci. Rep. 6, 22873 (2016).

[62] T. Ishikawa, A. Nakanishi, K. Shimizu, H. Katayama-Yoshida, T. Oda, and N. Suzuki, Sci. Rep. 6, 23160 (2016).

[63] X. Li, H. Liu, and F. Peng, Phys. Chem. Chem. Phys. 18, 28791 (2016).

[64] X. Zhong, H. Wang, J. Zhang, H. Liu, S. Zhang, H.-F. Song, G. Yang, L. Zhang, and Y. Ma, Phys. Rev. Lett. 116, 057002 (2016).

[65] Y. Fu, X. Du, L. Zhang, F. Peng, M. Zhang, C. J. Pickard, R. J. Needs, D. J. Singh, W. Zheng, and Y. Ma, Chem. Mater. 28, 1746 (2016).

[66] Y. Li, L. Wang, H. Liu, Y. Zhang, J. Hao, C. J. Pickard, J. R. Nelson, R. J. Needs, W. Li, Y. Huang et al., Phys. Rev. B 93, 020103(R) (2016).

[67] Y. Liu, D. Duan, F. Tian, C. Wang, Y. Ma, D. Li, X. Huang, B. Liu, and T. Cui, Phys. Chem. Chem. Phys. 18, 1516 (2016).

[68] A. Majumdar, J. S. Tse, M. Wu, and Y. Yao, Phys. Rev. B 96, 201107(R) (2017).

[69] H. Liu, I. I. Naumov, R. Hoffmann, N. W. Ashcroft, and R. J. Hemley, Proc. Natl. Acad. Sci. USA 114, 6990 (2017).

[70] M. M. D. Esfahani, A. R. Oganov, H. Niu, and J. Zhang, Phys. Rev. B 95, 134506 (2017).

[71] Q. Zeng, S. Yu, D. Li, A. R. Oganov, and G. Frappere, Phys. Chem. Chem. Phys. 19, 8236 (2017). 
[72] Q. Zhuang, X. Jin, T. Cui, Y. Ma, Q. Lv, Y. Li, H. Zhang, X. Meng, and K. Bao, Inorg. Chem. 56, 3901 (2017).

[73] T. Ishikawa, A. Nakanishi, K. Shimizu, and T. Oda, J. Phys. Soc. Jpn. 86, 124711 (2017).

[74] X. Li and F. Peng, Inorg. Chem. 56, 13759 (2017).

[75] X.-F. Li, Z.-Y. Hu, and B. Huang, Phys. Chem. Chem. Phys. 19, 3538 (2017).

[76] F. Peng, Y. Sun, C. J. Pickard, R. J. Needs, Q. Wu, and Y. Ma, Phys. Rev. Lett. 119, 107001 (2017).

[77] D. V. Semenok, I. A. Kruglov, A. G. Kvashnin, and A. R. Oganov, arXiv:1806.00865.

[78] D. V. Semenok, A. G. Kvashnin, I. A. Kruglov, and A. R. Oganov, J. Phys. Chem. Lett. 9, 1920 (2018).

[79] K. V. Shanavas, L. Lindsay, and D. S. Parker, Sci. Rep. 6, 28102 (2018).

[80] L. Wang, D. Duan, H. Yu, H. Xie, X. Huang, Y. Ma, F. Tian, D. Li, B. Liu, and T. Cui, Inorg. Chem. 57, 181 (2018).

[81] X. Ye, N. Zarifi, E. Zurek, R. Hoffmann, and N. W. Ashcroft, J. Phys. Chem. C 122, 6298 (2018).

[82] P. B. Allen and R. C. Dynes, Phys. Rev. B 12, 905 (1975).

[83] The predictor is as follows: $f\left(S, h, M, P, \mu^{*}\right)=\div \times+\mu^{*}-$ $-\div 9---8++\mu^{*}--\div 9-+\div \div 10102 \div$ $---\div \div \times 10+69 S-++6 h h M-\mu^{*} M+$ $\mu^{*} 101010 \times \div 824+6 h \times 4410 \div--+M 7+$ $+-9 \div---+-\div 9-\div P 6+6 h \times \times+$ $\mu^{*}+\mu^{*} \times \mu^{*} h 99+3 h \times 10+6 h 1010 P-9+$ $+S 7 M \div \div \times 87--\div P-+h 10--M 71-8+$

$$
\begin{aligned}
& ++81 \times \mu^{*} h \div--+M 7+\div \div \div+M 8 h- \\
& -+M++-\mu^{*} \times 9 h \div-\div \times-+-3 \times \\
& M M 104104-+69 \times 1 M P+\mu^{*} \times-\times-6 M \mu^{*}+ \\
& 1 \mu^{*} \div 991-+M 776++8 \div 75-9+\div 7 h S \times \\
& 1 M 6106 \times 1 M 6-\div 4--\div P+-541-8+ \\
& +3 h---\div 8-+\div \div 6102 \div--\times \times 4 h 1 \times \\
& 10+6 h M+M 7 \times \div 824 \times 10 \times \times 9 h M 1010 \div \\
& \times \times+\mu^{*}-\div \times+h 10104 \times M \times+69 \div 2 h 9 \times \\
& \div 10105 \times 4 M 10+6 h \times 9 h 109---9 \div-- \\
& -\div \times \times+\mu^{*} 109 \times \div--\div 9+\times \div M P 61 \times \\
& \times+\mu^{*}+\mu^{*} \times \mu^{*} h 9910105 \times 4 M \times 10 \times \div \div \\
& 9102 M 1010 P 9-\times h 99 .
\end{aligned}
$$

[84] H. Wang, J. S. Tse, K. Tanaka, T. Iitaka, and Y. Ma, Proc. Natl. Acad. Sci. USA 109, 6463 (2012).

[85] P. Giannozzi, S. Baroni, N. Bonini, M. Calandra, R. Car, C. Cavazzoni, D. Cereso, G. L. Chiarott, M. Cococcioni, I. Dabo et al., J. Phys.: Condens. Matter 21, 395502 (2009).

[86] J. P. Perdew, K. Burke, and M. Ernzerhof, Phys. Rev. Lett. 77, 3865 (1996).

[87] A. M. Rappe, K. M. Rabe, E. Kaxiras, and J. D. Joannopoulos, Phys. Rev. B 41, 1227 (1990).

[88] K. Momma and F. Izumi, J. Appl. Crystallogr. 44, 1272 (2011).

[89] A. Nakanishi, T. Ishikawa, and K. Shimizu, J. Phys. Soc. Jpn. 87, 124711 (2018).

[90] Y. Sun, J. Lv, Y. Xie, H. Liu, and Y. Ma, Phys. Rev. Lett. 123, 097001 (2019). 